PUPT-1421

September 1993

hep-th/9309106

\title{
Gravitational Dressing of Renormalization Group
}

\author{
I. R. Klebanov, I. I. Kogan ${ }^{\star}$ And A. M. Polyakov \\ Joseph Henry Laboratories \\ Princeton University \\ Princeton, New Jersey 08544
}

\begin{abstract}
We study the gravitational dressing of renormalizable two-dimensional field theories. Our main result is that the one-loop $\beta$-function is finitely renormalized by the factor $\frac{k+2}{k+1}$, where $k$ is the central charge of the gravitational $S L(2, R)$ current algebra.
\end{abstract}

\footnotetext{
$\star$ On leave of absence from ITEP, Moscow, Russia.
} 
In this letter we report on some minor progress in a very important subject the problem of gravitational dressing. It is well known that string theory in any dimension is described by two-dimensional field theory coupled to two-dimensional quantum gravity. This coupling leads to the physically important change of the scaling dimensions and to the appearance of unexpected symmetries [1, 2]. There are many important problems in string theory where one has to study the gravitational dressing of two-dimensional models which are renormalizable, but not conformally invariant. Here we will focus on precisely this new situation. More concretely, we will show how the renormalization group equation is deformed (in the one-loop approximation) by the gravitational dressing.

The Gell-Mann-Low $\beta$-function appears when one perturbs a conformal field theory by some marginal operators

$$
S=S_{0}+\sum_{n} \lambda_{n} \int O_{n}(x) d^{2} x
$$

The renormalization group equation for the coupling constants $\lambda_{n}$ has the form

$$
\frac{d \lambda_{n}}{d \log \Lambda}=\beta_{n}(\lambda)
$$

where $\Lambda$ is the ultraviolet cut-off.

It was realized long ago [3] that the Gell-Mann-Low $\beta$-function is related to the structure constants of the operator algebra. Namely, if we have an operator product expansion (OPE)

$$
O_{n}(x) O_{m}(0)=\frac{1}{|x|^{2}} g_{l}^{n m} O_{l}(0)+\ldots
$$

then

$$
\beta_{l}(\lambda)=2 \pi g_{l}^{n m} \lambda_{n} \lambda_{m}+\mathcal{O}\left(\lambda^{3}\right)
$$

In order to find these structure constants in any theory, it is sufficient to calculate the 2-point functions and the 3-point functions of the perturbing operators. Below we will 
perform this calculation in the novel situation where the two-dimensional quantum gravity is "turned on". Let us explain the precise meaning of this. In order to describe the effects of gravity it is advantageous to use the light-cone gauge of ref. [1], since the coordinate cut-off in this gauge is equivalent to the physical cut-off. The line element is $d s^{2}=2 d x^{+} d x^{-}+h_{++}(x)\left(d x^{+}\right)^{2}$, so that the gravitation is described by a field $h_{++}(x)$ coupled to the energy momentum tensor. Hence, "turning the gravity on" means replacing eq. (1) with the action

$$
S=S_{0}+\sum_{n} \lambda_{n} \int O_{n}(x) d^{2} x+\int h_{++}(x) T_{--}(x) d^{2} x
$$

where $T_{--}(x)$ is a component of the matter energy momentum tensor, and $h_{++}(x)$ is to be treated as a quantum field.

Without losing much generality, we will concentrate on the case where the marginal operators $O_{n}$ can be represented as products of right-moving and left-moving conserved currents. Thus, we chose the second term in (2) to be of the form

$$
\sum_{A, A^{\prime}} \lambda_{A A^{\prime}} \int d^{2} x J_{-}^{A}\left(x^{-}\right) J_{+}^{A^{\prime}}\left(x^{+}\right)
$$

The 2-point and 3-point functions of the currents are

$$
\begin{aligned}
& <J_{-}^{A}\left(x_{1}\right) J_{-}^{B}\left(x_{2}\right)>=K \delta^{A B} \frac{1}{\left(x_{1}^{-}-x_{2}^{-}\right)^{2}}, \\
& <J_{-}^{A}\left(x_{1}\right) J_{-}^{B}\left(x_{2}\right) J_{-}^{C}\left(x_{3}\right)>=f^{A B C} \frac{1}{\left(x_{1}^{-}-x_{2}^{-}\right)\left(x_{2}^{-}-x_{3}^{-}\right)\left(x_{3}^{-}-x_{1}^{-}\right)},
\end{aligned}
$$

and similarly for $J_{+}^{A^{\prime}}$. In this case the structure constants of the OPE are the products of the structure constants of the current algebra,

$$
g_{\left(A A^{\prime}\right)}^{\left(B B^{\prime}\right)\left(C C^{\prime}\right)}=f_{A}^{B C} f_{A^{\prime}}^{B^{\prime} C^{\prime}}
$$

where $f_{A}^{B C}=\frac{1}{K} \delta_{A D} f^{D B C}$. 
Now, let us consider the effects of the gravitational dressing. In the light-cone gauge, the correlation functions of the $J_{+}^{A^{\prime}}$ are unchanged by gravity, while those of the $J_{-}^{A}$ are renormalized, so that the first factor in eq. (4) changes. In order to calculate this effect, we need to perform the following functional integral,

$\ll J_{-}^{A_{1}}\left(x_{1}\right) \ldots J_{-}^{A_{n}}\left(x_{n}\right) \gg=\int\left[D h_{++}(x)\right]<J_{-}^{A_{1}}\left(x_{1}\right) \ldots J_{-}^{A_{n}}\left(x_{n}\right) e^{i \int h_{++}(x) T_{--}(x) d^{2} x}>$

where the double brackets denote the gravitationally dressed correlators. This path integral is calculable essentially because the Ward identities determine the correlation functions containing any number of insertions of $T_{--}$. As a result, the dressed correlator (5) satisfies a rather peculiar differential equation of the type derived in ref. [1]. This equation is derived from the conservation law for the dressed currents,

$$
\partial_{+} J_{-}^{A}=\partial_{-}\left(h_{++} J_{-}^{A}\right)
$$

which comes from the gauge-fixed version of $D^{\beta} J_{\beta}^{A}=0$. The product $h_{++} J_{-}^{A}$ has to be properly regularized. It can be calculated explicitly because the Ward identities determine the correlation functions of $h_{++}$. In particular, we have [1]

$$
\begin{aligned}
& \ll h_{++}(w) J^{B_{1}}\left(x_{1}\right) J^{B_{2}}\left(x_{2}\right) \ldots J^{B_{n}}\left(x_{n}\right) \gg= \\
& -\frac{1}{k+2} \sum_{i=1}^{n} \frac{\partial}{\partial x_{i}^{-}} \frac{\left(w^{-}-x_{i}^{-}\right)^{2}}{w^{+}-x_{i}^{+}} \ll J^{B_{1}}\left(x_{1}\right) J^{B_{2}}\left(x_{2}\right) \ldots J^{B_{n}}\left(x_{n}\right) \gg,
\end{aligned}
$$

where $k$ is the central charge of the gravitational $S L(2, R)$ current algebra, related to the matter central charge by the formula [2]

$$
k+2=\frac{1}{12}(c-13 \pm \sqrt{(c-1)(c-25)})
$$

In order to match onto the semiclassical limits $|c| \rightarrow \infty$, the + sign is chosen for $c \geq 25$, and the - sign for $c \leq 1$. Combining eqs. (6) and (7), we obtain the following 
differential equation,

$$
\begin{aligned}
& \frac{\partial}{\partial w^{+}} \ll J_{-}^{A}(w) J_{-}^{B_{1}}\left(x_{1}\right) J_{-}^{B_{2}}\left(x_{2}\right) \ldots J_{-}^{B_{n}}\left(x_{n}\right) \gg= \\
& -\frac{1}{k+2} \sum_{i=1}^{n} \frac{\partial^{2}}{\partial w^{-} \partial x_{i}^{-}} \frac{\left(w^{-}-x_{i}^{-}\right)^{2}}{w^{+}-x_{i}^{+}} \ll J_{-}^{A}(w) J_{-}^{B_{1}}\left(x_{1}\right) J_{-}^{B_{2}}\left(x_{2}\right) \ldots J_{-}^{B_{n}}\left(x_{n}\right) \gg \\
& -2 \pi i \sum_{i=1}^{n} \frac{\partial}{\partial w^{-}} \delta^{2}\left(w-x_{i}\right) K \delta^{A B_{i}} \ll J_{-}^{B_{1}}\left(x_{1}\right) \ldots J_{-}^{B_{i-1}}\left(x_{i-1}\right) J_{-}^{B_{i+1}}\left(x_{i+1}\right) \ldots J_{-}^{B_{n}}\left(x_{n}\right) \gg \\
& +2 \pi i \sum_{i=1}^{n} \delta^{2}\left(w-x_{i}\right) f_{C}^{A B_{i}} \ll J_{-}^{B_{1}}\left(x_{1}\right) \ldots J_{-}^{B_{i-1}}\left(x_{i-1}\right) J_{-}^{C}\left(x_{i}\right) J_{-}^{B_{i+1}}\left(x_{i+1}\right) \ldots J_{-}^{B_{n}}\left(x_{n}\right) \gg
\end{aligned}
$$

where $K$ is the central charge of the flat space current algebra, and $f^{A B C}$ are its structure constants from eq. (3). Note that the first term on the right-hand side of eq. (9) can be regarded as the gravitational violation of the right-moving nature of the current, while the remaining delta-function terms are the violations that take place even without the coupling to gravity. It is an interesting exercise to check eq. (9) in the $1 / c$ expansion directly from the path integral (5).

Eqs. (9) in principle solve the problem of gravitational dressing. We suspect that they contain many mathematical and physical surprises. Presently we will make only a limited use of them. Namely, we determine the 2-point and the 3-point functions, and thus solve the problem of gravitational dressing for the one-loop renormalization group $\beta$-function. The key point is that the $x$-dependence in these two cases is completely fixed by the projective invariance,

$$
\begin{aligned}
& \ll J_{-}^{A}\left(x_{1}\right) J_{-}^{B}\left(x_{2}\right) \gg=\tilde{K} \delta^{A B} \frac{1}{\left(x_{1}^{-}-x_{2}^{-}\right)^{2}} \\
& \ll J_{-}^{A}\left(x_{1}\right) J_{-}^{B}\left(x_{2}\right) J_{-}^{C}\left(x_{3}\right) \gg=\tilde{f}^{A B C} \frac{1}{\left(x_{1}^{-}-x_{2}^{-}\right)\left(x_{2}^{-}-x_{3}^{-}\right)\left(x_{3}^{-}-x_{1}^{-}\right)} .
\end{aligned}
$$

In order to determine the constants $\tilde{K}$ and $\tilde{f}^{A B C}$, we substitute the ansatz (10) into eqs. (9). From the equation for the 2-point function, we find

$$
\tilde{K}=K-\frac{1}{k+2} \tilde{K}
$$


From the equation for the 3-point function, we obtain a relation

$$
\tilde{f}^{A B C}=\frac{\tilde{K}}{K} f^{A B C}+\frac{1}{k+2} \tilde{f}^{A B C}
$$

Solving eqs. (11) and (12), we find

$$
\begin{aligned}
& \tilde{K}=\frac{k+2}{k+3} K \\
& \tilde{f}^{A B C}=f^{A B C} \frac{(k+2)^{2}}{(k+1)(k+3)} .
\end{aligned}
$$

Since the indices are now raised and lowered with the metric given by the two-point function in eq. (10), we have

$$
\tilde{f}_{A}^{B C}=\frac{1}{\tilde{K}} \delta_{A D} \tilde{f}^{D B C}=\frac{1}{K} \delta_{A D} f^{D B C} \frac{k+2}{k+1}
$$

Recalling that the structure constants are given by eq. (4), we have

$$
\tilde{g}_{\left(A A^{\prime}\right)}^{\left(B B^{\prime}\right)\left(C C^{\prime}\right)}=\frac{k+2}{k+1} g_{\left(A A^{\prime}\right)}^{\left(B B^{\prime}\right)\left(C C^{\prime}\right)}
$$

i.e. the one-loop $\beta$-function is multiplicatively renormalized by the gravitational dressing. This universal "renormalization of the renormalization group" constitutes our main result.

In order to further investigate the gravitational dressing of the renormalization group flow, we need to calculate the higher-order terms in the $\beta$-function. The first such term, which is $\mathcal{O}\left(\lambda^{3}\right)$, is determined by the 4-point function of the currents. Here the calculation is more complicated because the $x$-dependence is not completely fixed. Instead, the projective invariance requires that

$$
\ll J_{-}^{A}(w) J_{-}^{B}(z) J_{-}^{C}(x) J_{-}^{D}(y) \gg=\frac{F^{A B C D}(t, \bar{t})}{(x-y)^{2}(z-w)^{2}},
$$


where

$$
t=\frac{(w-x)(z-y)}{(w-y)(z-x)}
$$

After substituting this form into eq. (9) for the 4-point function, we find

$$
-(k+2) \bar{t} \frac{\partial F}{\partial \bar{t}}=2 F \frac{t+1}{t-1}-3 t \frac{\partial F}{\partial t}+(\bar{t}-t) \frac{t-1}{\bar{t}-1} \frac{\partial}{\partial t}\left(t \frac{\partial F}{\partial t}\right)
$$

The boundary conditions on $F$ at the special points $t=0,1$ and $\infty$ are determined by the singular terms in eq. (9). Eqn. (16) has a rich variety of solutions. Perturbation theory in $1 / c$ suggests that there is only one physically acceptable solution. Let us consider, for instance, a theory of $c$ free massless Dirac fermions, where the currents are represented as $J_{-}^{A}=\psi_{-j}^{\dagger} \lambda_{j i}^{A} \psi_{-i}$, and $\lambda^{A}$ are generators of $S U(N)$. Here the 4point function is of the form $(15)$, and $F^{A B C D}(t, \bar{t})$ is a sum of terms distinguished by their $S U(N)$ structure. There are two types of contributions:

$$
\delta^{A B} \delta^{C D}\left(1-\frac{12}{c} \frac{t+1}{t-1} \log |t|^{2}+\mathcal{O}\left(1 / c^{2}\right)\right)
$$

plus two other $\delta \cdot \delta$ terms, and

$$
\operatorname{Tr}\left(\lambda^{A} \lambda^{B} \lambda^{C} \lambda^{D}\right)\left[\left(1+\frac{12}{c}\right) \frac{t-1}{t}-\frac{6}{c}\left(\log |t|^{2}+\frac{(t-1)^{2}}{t^{2}} \log |t-1|^{2}\right)+\mathcal{O}\left(1 / c^{2}\right)\right]
$$

plus five other terms of this type. The correct solution should be chosen to match these perturbative results. Let us also note that the acquired dependence of the 4point function on $\bar{t}$ is a purely gravitational effect that is probably connected with the appearance of new states, the gravitational descendants.

We have found our main result, eq. (14), using the light-cone gauge for the twodimensional quantum gravity. We believe, however, that this result is completely

\footnotetext{
$\star$ Here we find it convenient to continue to the Euclidean signature so that $x^{-}$is replaced by a complex variable $x$, and $x^{+}$- by its complex conjugate $\bar{x}$.
} 
universal and gauge independent. In order to illustrate how a similar calculation proceeds in the conformal gauge, we will study the gravitational dressing of the $O(N)$ model. The model is defined by the action

$$
S=\frac{1}{2 e^{2}} \int d^{2} x \sqrt{g} g^{\mu \nu} \partial_{\mu} \vec{n} \cdot \partial_{\nu} \vec{n},
$$

where $\vec{n}$ is an $N$-component vector of length 1 . If we choose the conformal gauge [4] $g_{\mu \nu}=e^{-\phi} \delta_{\mu \nu}$, then $\phi$ becomes dynamical and can be regarded as an extra coordinate of critical string theory. We will assume that the target space metric in this theory is of the form

$$
d s^{2}=d \phi^{2}+a^{2}(\phi) d \Omega^{2}
$$

where $d \Omega^{2}$ is the metric of a unit sphere in $N$ dimensions. We also need to introduce the dilaton field $\Phi(\phi)$, which couples to the world sheet curvature [5]. The variable $\phi$ defines the world sheet scale, and the effective radius of the sphere becomes scaledependent through quantum effects. The form of this dependence is determined by the conformal invariance or, equivalently, by the consistency of the critical string. In the one-loop approximation, the consistency equations are well-known to be [6]

$$
\begin{aligned}
& R_{i j}=\nabla_{i} \nabla_{j} \Phi, \\
& \nabla^{2} \Phi+(\nabla \Phi)^{2}=\frac{26-N}{3} .
\end{aligned}
$$

Substituting our ansatz for $G_{i j}$ and $\Phi$ into these equations, we arrive at

$$
\begin{aligned}
& N-2-a a^{\prime \prime}-(N-2)\left(a^{\prime}\right)^{2}=a a^{\prime} \Phi^{\prime}, \\
& \Phi^{\prime \prime}+(N-1) \frac{a^{\prime \prime}}{a}=0, \\
& \Phi^{\prime \prime}+(N-1) \frac{a^{\prime}}{a} \Phi^{\prime}+\left(\Phi^{\prime}\right)^{2}=\frac{26-N}{3} .
\end{aligned}
$$

For large $\phi$, the asymptotic form of the needed solution to these equations is

$$
\begin{aligned}
& \Phi=Q \phi+\mathcal{O}(\log \phi), \\
& a^{2}=2 \frac{N-2}{Q} \phi+\mathcal{O}(\log \phi),
\end{aligned}
$$


where $Q=\sqrt{\frac{25-c}{3}}$ and $c=N-1$. In order to calculate the gravitationally dressed $\beta$-function, we need a precise definition of the physical scale. A sensible definition is to identify the scale $\Lambda^{-2}$ with the cosmological constant operator, also known as the "tachyon background" $T(\phi){ }^{\star}$ Its leading asymptotic for large $\phi$ is [7]

$$
\begin{aligned}
& \Lambda^{-2} \sim T(\phi) \sim e^{-\alpha \phi}, \\
& \alpha=\frac{\sqrt{25-c}-\sqrt{1-c}}{\sqrt{12}} .
\end{aligned}
$$

Thus, the gravitationally dressed renormalization group equation is

$$
\frac{d a^{2}}{d \log \Lambda}=2(N-2) \frac{2}{Q \alpha}+\mathcal{O}\left(a^{-2}\right)
$$

The form of the equation before coupling to gravity is well known to be

$$
\frac{d a^{2}}{d \log \Lambda}=2(N-2)+\mathcal{O}\left(a^{-2}\right)
$$

where $a^{2}=4 \pi / e^{2}$. Therefore, the gravitational renormalization factor for the oneloop $\beta$-function is $\frac{2}{Q \alpha}$ which, with eq. (8), easily reduces to $\frac{k+2}{k+1}$. This provides a non-trivial check of the universality of our results.

We suspect that there are similar universal gravitational renormalizations for the sub-leading terms in the $\beta$-function. The problem of determination of the full gravitational effect on the renormalization group is a fascinating challenge.

Acknowledgements:

The research of I. R. K. is supported in part by DOE grant DE-AC02-76WRO3072, NSF Presidential Young Investigator Award PHY-9157482, James S. McDonnell Foundation grant No. 91-48, and an A.P. Sloan Foundation Research Fellowship. The research of A. M. P. and I. I. K. is supported in part by the NSF grant PHY90-21984.

$\star$ A good test of this definition is the eventual agreement of the one-loop $\beta$-function with the light-cone gauge calculation. It is possible, however, that the correct definition of scale is more subtle for higher-order calculations in the conformal gauge. 


\section{REFERENCES}

1. A. M. Polyakov, Mod. Phys. Lett. A2 (1987) 893.

2. V. Knizhnik, A. Polyakov and A. Zamolodchikov, Mod. Phys. Lett. A3 (1988) 819.

3. A. M. Polyakov, ZhETF 63 (1972) 24.

4. A. M. Polyakov, Phys. Lett. 103 B (1981) 207.

5. E. S. Fradkin and A. A. Tseytlin, Phys. Lett. 158 B (1985) 316.

6. C. Callan, E. Martinec, M. Perry and D. Friedan, Nucl. Phys. B262 (1985) 593.

7. F. David, Mod. Phys. Lett. A3 (1988) 1651; J. Distler and H. Kawai, Nucl. Phys. B321 (1989) 509. 